\title{
INFLUÊNCIA DA TEMPERATURA DE ARMAZENAMENTO NA QUALIDADE DAS SEMENTES DE Caesalpinia peltophoroides Benth. (SIBIPIRUNA) ${ }^{1}$
}

\author{
Claudia Aparecida Pontes², Viviana Borges Corte², Eduardo Euclydes de Lima e Borges ${ }^{3}$ Aderlan Gomes \\ da Silva ${ }^{4}$ e Rita de Cássia Gonçalves Borges ${ }^{3}$
}

\begin{abstract}
RESUMO - Este trabalho teve como objetivo avaliar o efeito da temperatura de armazenamento na porcentagem de germinação e no vigor das sementes de Caesalpinia peltophoroides Benth. (sibipiruna), por meio de testes fisiológicos. O teor de água aumentou nas sementes armazenadas a $5{ }^{\circ} \mathrm{C}$ e reduziu naquelas a $20^{\circ} \mathrm{C}$. A porcentagem de germinação das sementes mantidas a 5 e $20^{\circ} \mathrm{C}$ apresentou redução aos 220 e 120 dias, respectivamente. O índice de velocidade de germinação decresceu de maneira similar. A condutividade elétrica não foi alterada nas sementes armazenadas a $5{ }^{\circ} \mathrm{C}$ e aumentou nas que permaneceram a $20^{\circ} \mathrm{C}$. O envelhecimento acelerado nos períodos de 24, 48 e 72 horas detectou redução significativa na qualidade das sementes em ambos os ambientes de armazenamento. A redução da porcentagem de germinação e do vigor foi maior nas sementes armazenadas a $20^{\circ} \mathrm{C}$.
\end{abstract}

Palavras-chave: Caesalpinia peltophoroides, germinação, vigor e armazenamento.

\section{INFLUENCE OF STORAGE TEMPERATURE ON SEED QUALITY OF Caesalpinia peltophoroides Benth.}

\begin{abstract}
The aim of this work was to evaluate the effect of storage temperature on the percentage of seed germination and vigor of Caesalpinia peltophoroides (Caesalpiniaceae) seeds through physiological tests. The water content increased in seeds stored at $5^{\circ} \mathrm{C}$ and decreased in seeds stored at $20^{\circ} \mathrm{C}$. The percentage of germination of seeds stored at 5 and $20^{\circ} \mathrm{C}$ was reduced from 220 and 120 days, respectively. The germination velocity index decreased in a similar way. The electrical conductivity was not altered on seeds stored at $5^{\circ} \mathrm{C}$ and increased on those kept at $20^{\circ} \mathrm{C}$. The accelerated aging for periods of 24,48 and 72 hours markedly reduced seed quality in both storage environments. The reduction on percentage of seed germination and vigor was grater on seeds stored at $20^{\circ} \mathrm{C}$.
\end{abstract}

Keywords: Caesalpinia peltophoroides, temperature, storage, germination and vigor.

\section{INTRODUÇÃO}

Caesalpinia peltophoroides (sibipiruna) é uma espécie ornamental e com potencial madeireiro. No Brasil, ocorre principalmente na região de Mata Atlântica do Rio de Janeiro, sul da Bahia e no Pantanal MatoGrossense. Sua madeira é pesada, dura e de média durabilidade, sendo utilizada na construção civil e na produção de móveis em geral. Pode ser utilizada em plantios mistos para recuperação de áreas degradas e, principalmente, no paisagismo. A espécie é pouco exigente com relação ao tipo de solo. A árvore é semidecídua e heliófila, produzindo anualmente grande quantidade de sementes (LORENZI, 1992).

\footnotetext{
${ }^{1}$ Recebido em 21.07.2004 e aceito para publicação em 10.11.2005.

${ }^{2}$ Programa de Pós-Graduação em Ciência Florestal. E-mail: <pontesac@gmail.com

${ }^{3}$ Departamento de Engenharia Florestal da Universidade Federal de Viçosa - DEF/UFV, 36570-000 Viçosa-MG.

${ }^{4}$ Program de Pós-Graduação em Fitopatologia da UFV.
} 
A deterioração é um dos grandes problemas do armazenamento de sementes, principalmente das oleaginosas (BRACCINI et al., 2001). A deterioração ocorre em níveis molecular, genético, celular, de tecido e de população da semente (MATTHEWS, 1985). O conhecimento dessas mudanças serve de base para elucidação dos mecanismos de envelhecimento, como também para o aprimoramento de métodos para avaliação do vigor. A deterioração não pode ser evitada, mas pode ser controlada, sendo esse o principal objetivo do armazenamento, que está entre as estratégias de conservação ex situ mais utilizadas, por preservar as características genéticas das sementes até que sejam semeadas (CARNEIRO,1985; NODARI et al., 1998).

Sementes de sibipiruna, quando armazenadas em câmara fria, mantém sua capacidade germinativa por 360 dias (FIGLIOLIA et al., 2001), com a ressalva de que sementes armazenadas com baixo teor de água se conservaram melhor (FIGLIOLIA, 1988).

Diante da importância da espécie, este trabalho teve como objetivo avaliar o efeito da temperatura de armazenamento na porcentagem de germinação e no vigor das sementes de Caesalpinia peltophoroides Benth. (sibipiruna).

\section{MATERIAL E MÉTODOS}

As sementes de sibipiruna foram colhidas de três indivíduos adultos no Campus da Universidade Federal de Viçosa, entre julho e agosto de 2002. As sementes foram acondicionadas em caixas de papelão ( $25 \times 22$, $5 \mathrm{~cm}$ ) e armazenadas a $5^{\circ} \mathrm{C}$ e $70 \%$ de umidade relativa e a $20^{\circ} \mathrm{C}$ e $62 \%$ de umidade relativa. As avaliações foram realizadas em intervalos de 60 dias até 180 dias, tendo sido a última avaliação realizada aos 220 dias de armazenamento. Procedeu-se à quantificação do teor de água e à condução de testes fisiológicos de germinação, de envelhecimento acelerado e de condutividade elétrica. As avaliações realizadas antes do armazenamento serviram como testemunha (tratamento adicional).

O teor de água foi determinado pelo método de estufa a $105 \pm 3^{\circ} \mathrm{C}$, durante 24 horas (BRASIL, 1992). Para a condução do teste de germinação, todas as sementes passaram por um processo de desinfestação com o fungicida Captan 0,5\%. Em seguida, as sementes foram colocadas em placa de Petri sobre duas folhas de papel-filtro, tipo germitest, umedecidas com água destilada. Permanecendo em germinador, à temperatura constante de $25^{\circ} \mathrm{C}$, com luz contínua, fornecida por lâmpadas fluorescentes de 40 watts, tipo luz do dia, por um período de 10 dias. Foram feitas avaliações diárias, sendo consideradas germinadas as sementes que emitiram radícula. O índice de velocidade de germinação foi calculado com a fórmula proposta por Maguire (1962). A condutividade elétrica foi avaliada conforme o procedimento descrito por Woodstock (1973) e medida em condutivímetro MICRONAL, modelo B 330 , eletrodo com constante 0,75 . O envelhecimento acelerado foi realizado segundo a metodologia descrita por Marcos Filho (1994). Após o período de envelhecimento, foi realizado teste de germinação e calculados a porcentagem e a velocidade de germinação, conforme descrito anteriormente.

O delineamento experimental utilizado foi o inteiramente casualizado, no esquema fatorial $4 \times 2$ (tempo $\mathrm{x}$ temperatura), com tratamento adicional (testemunha), tendo cinco repetições de 20 sementes cada, nas variáveis germinação, índice de velocidade de germinação, condutividade elétrica e três repetições de 20 sementes, para a variável teor de água. Para a normalização das variáveis, os valores de porcentagem e índice de velocidade de germinação foram transformados pela função Arco-seno $\sqrt{x / 100}$; a variável porcentagem de germinação, após o envelhecimento acelerado, foi transformada pela função $\sqrt{x+0,5}$ (BANZATTO e KRONKA, 1989). Os dados de condutividade elétrica e de teor de água não foram transformados.

Todas as variáveis foram submetidas à análise de variância seguida de regressão, considerando-se o tratamento adicional (GARCIA, 2001). Para comparar o efeito da temperatura $\left(5\right.$ e $20^{\circ} \mathrm{C}$ ) foram realizados testes de identidade de modelos, para verificar paralelismo, concorrência e, ou, coincidência. O teste de Dunnett foi aplicado quando houve significância entre as interações fatorial e testemunha. As análises foram realizadas em nível $\alpha$ de 5\% de significância. Os gráficos foram construídos com os dados originais. As análises descritas foram realizadas utilizando-se o programa Microsoft Excel ${ }^{\circledR}$ e o programa Statistica 5.5 .

\section{RESULTADOS E DISCUSSÃO}

O teor de água das sementes de Caesalpinia peltophoroides foi significativamente diferente entre os dois ambientes de armazenamento, por meio do teste 
de identidade de modelo. As sementes mantidas a 5 ${ }^{\circ} \mathrm{C}$ apresentaram aumento no teor de água em relação à testemunha, enquanto aquelas em $20^{\circ} \mathrm{C}$, redução (Figura 1). Trabalhando com a mesma espécie, Figliolia (1988) constatou que as sementes armazenadas em ambiente de câmara fria e com menor teor de água (8,5\%) mantiveram a capacidade germinativa por até 240 dias. Em outro trabalho, Figliolia et al. (2001) observaram que a capacidade germinativa das sementes de sibipiruna se manteve por 360 dias, quando estas foram armazenadas em câmara fria, e a variação no teor de água foi baixo. Segundo Varela e Façanha (1987), baixos níveis de umidade reduzem a taxa respiratória e contribuem para a redução do metabolismo das sementes, resultando em maior vigor e viabilidade. León-Lobos e Ellis (2002) estudaram a viabilidade das sementes de Fagus sylvatica e F. crenata durante o armazenamento, observando redução significativa e progressiva da germinação com a redução do teor de água de 14 para 3\%. O melhor ambiente de armazenamento foi a $-10^{\circ} \mathrm{C}$ e teor de água de 7,8 a 11,5\% para Fagus sylvatica e de $-20^{\circ} \mathrm{C}$ e teor de água de 9,5 para Fagus crenata.

As sementes de Caesalpinia peltophoroides armazenadas a $5{ }^{\circ} \mathrm{C}$ mantiveram a porcentagem de germinação constante até 220 dias, enquanto a $20^{\circ} \mathrm{C}$ a redução foi detectada a partir dos 120 dias de armazenamento, pelo teste de Dunnett $(\mathrm{p}<0,05)$ (Figura 2a).

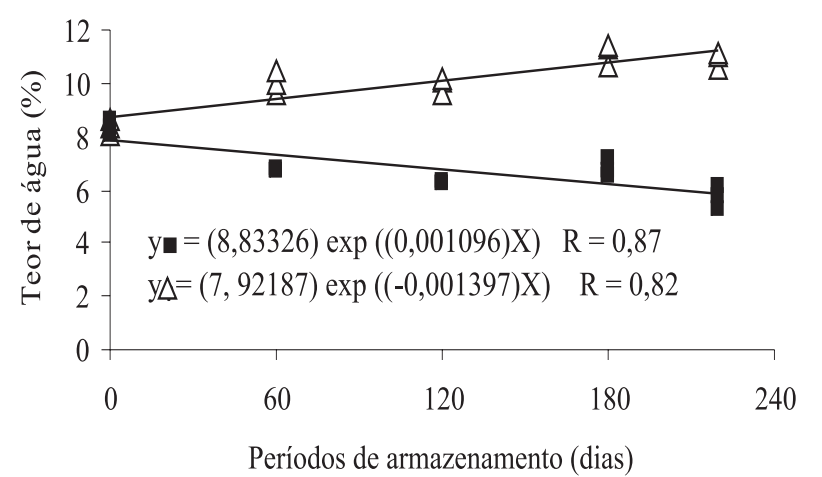

Figura 1 - Teor de água (\%) em sementes de Caesalpinia peltophoroides (Benth.), em função do período de armazenamento. $\mathrm{y}_{\mathbf{m}}$ teor de água a $20^{\circ} \mathrm{C}$ e $\mathrm{y}_{\wedge}$ teor de água a $5{ }^{\circ} \mathrm{C}$.

Figure 1 - Water content (\%) on Caesalpinia peltophoroides (Benth.) seeds as a function of storage period. $y_{n}$ water content at $20^{\circ} \mathrm{C}, y \wedge$ water content at $5^{\circ} \mathrm{C}$.
Por meio do teste de identidade de modelo, foi possível detectar diferença significativa na qualidade das sementes entre os dois ambientes de armazenamento. O índice de velocidade de germinação das sementes armazenadas a $5{ }^{\circ} \mathrm{C}$ foi maior do que o daquelas armazenadas a $20^{\circ} \mathrm{C}$ (Figura $2 b$ ). Diante dos resultados, a porcentagem de germinação e o índice de velocidade de germinação se mostraram adequados para avaliar a qualidade das sementes de sibipiruna, no período de realização do experimento.

Firmino et al. (1995) constataram que os testes de germinação e o cálculo da velocidade de germinação não se mostraram adequados para a avaliação da qualidade fisiológica das sementes de Amburana acreana (cerejeira). Resultado semelhante foi obtido por Freitas et al. (2001), que não encontraram relação significativa para a avaliação da qualidade fisiológica das sementes de Gossypium hirsutum, através dos testes de germinação.

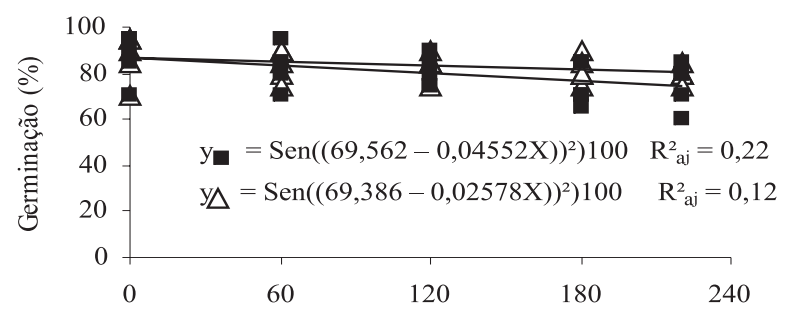

a) Períodos de armazenamento (dias)

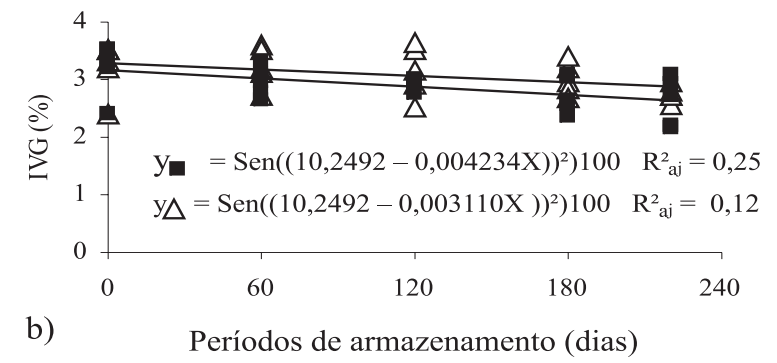

Figura 2-Porcentagem de germinação (a) e índice de velocidade de germinação (IVG) (b) de sementes de Caesalpinia peltophoroides (Benth.), em função do período de armazenamento. y. germinação (\%) a $20^{\circ} \mathrm{C}, \mathrm{y}_{\wedge}$ germinação a $5^{\circ} \mathrm{C}$. yø IVG $(\%)$ a $20^{\circ} \mathrm{C}$ e y^ IVG (\%) a $5{ }^{\circ} \mathrm{C}$.

Figure 2 - Seed germination (a) and germination velocity index (b) of Caesalpinia peltophoroides (Benth.) seeds as a function of storage period. $y_{\square}$ germination (\%) at $20^{\circ} \mathrm{C}, y_{\wedge}$ germination at $5{ }^{\circ} \mathrm{C} . y_{\mathbf{}} I V G$ (\%) at $20^{\circ} \mathrm{C}, y_{\wedge} I V G(\%)$ at $5{ }^{\circ} \mathrm{C}$.

R. Árvore, Viçosa-MG, v.30, n.1, p.43-48, 2006 
Esses mesmos autores enfatizaram a importância de se usar mais de um teste na avaliação de vigor.

A condutividade elétrica foi constante nas sementes mantidas a $5{ }^{\circ} \mathrm{C}$, e naquelas que permaneceram no ambiente de $20^{\circ} \mathrm{C}$ houve aumento significativo $(\mathrm{p}<0,05)$ ao longo do período de armazenamento (Figura 3 ). Provavelmente, na temperatura de $5{ }^{\circ} \mathrm{C}$ o processo de embebição tenha sido mais lento, com as sementes apresentando maior teor de água, em torno de 9\% (Figura 1), ou seja, maior organização das membranas celulares. No ambiente de $20^{\circ} \mathrm{C}$, a embebição foi mais rápida devido ao baixo teor de água (6\%), que pode resultar em maiores danos às membranas. A temperatura de $20{ }^{\circ} \mathrm{C}$ pode estar contribuindo para o processo de deterioração das sementes de Caesalpinia peltophoroides. Borges et al. (1992) não detectaram alteração significativa na condutividade elétrica em sementes de Piptadenia communis submetidas ao envelhecimento acelerado, concluindo que o processo de embebição reduziu os danos às membranas.

As sementes de Caesalpinia peltophoroides submetidas ao envelhecimento acelerado por 24, 48 e 72 horas apresentaram redução significativa $(p<0,05)$ na porcentagem de germinação com relação ao tempo de armazenamento, independentemente da temperatura

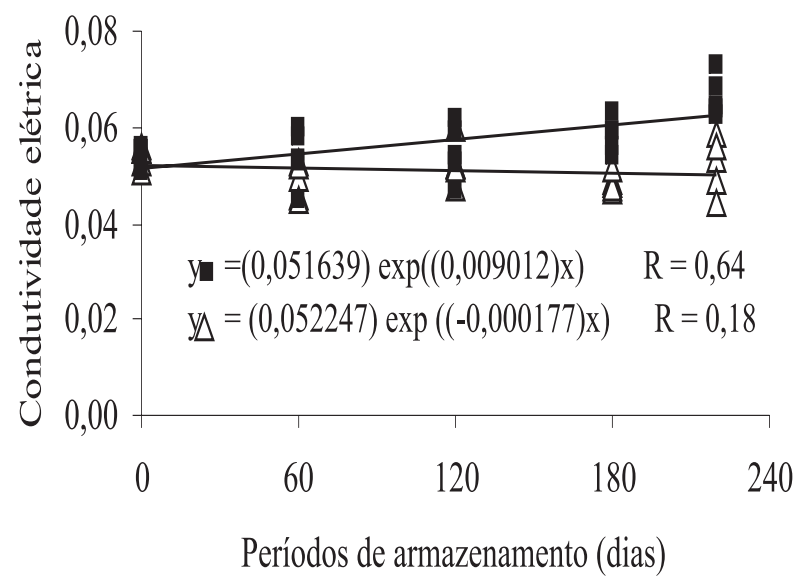

Figura 3 - Condutividade elétrica $(\mathrm{mS} / \mathrm{cm} / \mathrm{g})$ em sementes de Caesalpinia peltophoroides (Benth.), em função do período de armazenamento. $\mathrm{y}_{\Perp}$ Condutividade elétrica a $20^{\circ} \mathrm{C}$ e $y_{\wedge}$ Condutividade elétrica a $5^{\circ} \mathrm{C}$.

Figure 3 - Electrical conductivity $(\mathrm{mS} / \mathrm{cm} / \mathrm{g}$ ) on Caesalpinia peltophoroides (Benth.) seeds as a function of storage period. $y_{\square}$ Electrical conductivity at $20^{\circ} \mathrm{C}, y_{\Lambda}$ Electrical conductivity at $5{ }^{\circ} \mathrm{C}$.

R. Árvore, Viçosa-MG, v.30, n.1, p.43-48, 2006
(5 e $20^{\circ} \mathrm{C}$ ) (Figura 4a,b,c). No período de 24 horas de envelhecimento, essa redução significativa $(p<0,05)$ ocorreu a partir de 180 e 60 dias de armazenamento, nas temperaturas de 5 e $20^{\circ} \mathrm{C}$, respectivamente (Figura 4a). Os períodos de envelhecimento de 48 horas (Figura 4b) e 72 horas (Figura 4c) apresentaram redução significativa $(\mathrm{p}<0,05)$ na porcentagem de germinação a partir dos 60 dias de armazenamento, em ambas as temperaturas.

O índice de velocidade de germinação apresentou comportamento semelhante ao da porcentagem de germinação no mesmo período de envelhecimento, tendo tido redução significativa $(\mathrm{p}<0,05)$ do índice quando envelhecida por 24, 48 e 72 horas, a partir dos 60 dias de armazenamento (Figura 4d,e,f).

As sementes de Caesalpinia peltophoroides submetidas ao envelhecimento acelerado apresentaram redução de sua qualidade com o aumento do tempo de envelhecimento, provavelmente devido aos danos causados às membranas celulares.

Gemaque (1999), trabalhando com sementes de Tabebuia impetiginosa, observou o aumento da germinação e do índice de velocidade de germinação com 96 horas de envelhecimento, concluindo que houve processo de reestruturação do sistema de membrana. Em outro estudo, Borges et al. (1992) observaram redução da viabilidade em sementes Piptadenia communis submetidas ao envelhecimento acelerado, tendo sido a velocidade de germinação maior nas sementes envelhecidas por 16 e 20 horas. Segundo esses mesmos autores, o processo de pré-embebição pode ter favorecido a germinação.

\section{CONCLUSÕES}

Por meio dos testes fisiológicos, foi possível detectar a influência significativa da temperatura de armazenamento na porcentagem de germinação e no vigor das sementes de sibipiruna. No período testado, a temperatura de $5{ }^{\circ} \mathrm{C}$ foi a mais indicada para a manutenção da qualidade das sementes.

\section{AGRADECIMENTOS}

Ao CNPq, pela concessão da bolsa de mestrado. 


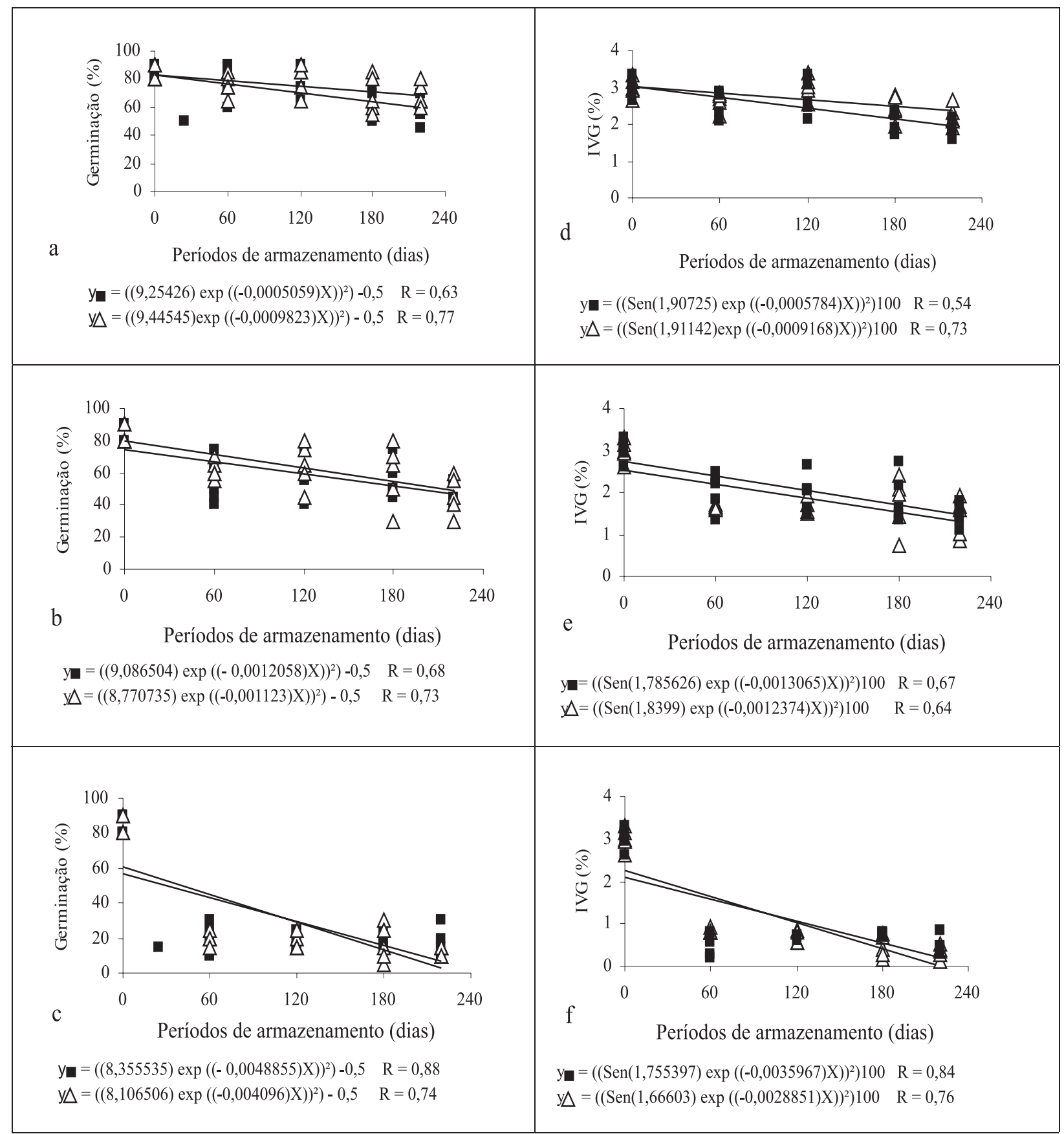

Figura 4 - Porcentagem de germinação (a, b e c) e índice de velocidade de germinação (IVG) (d, e e f) de sementes de Caesalpinia peltophoroides (Benth.) submetidas ao envelhecimento acelerado por 24, 48 e 72 horas, em função do período de armazenamento. yø germinação (\%) a $20^{\circ} \mathrm{C}, \mathrm{y}_{\wedge}$ germinação a $5{ }^{\circ} \mathrm{C}, \mathrm{y}_{\Perp} \mathrm{IVG}(\%)$ a $20^{\circ} \mathrm{C}$ e $\mathrm{y}_{\wedge}$ IVG a $5^{\circ} \mathrm{C}$.

Figure 4-Seed germination (Figures $a, b, c$ ) and germination velocity index (Figures d, e, f) of Caesalpinia peltophoroides (Benth.) seeds subjected to accelerated aging for 24,48 and 72 hours as a function of storage period. $y_{\square}$ Germination (\%) at $20^{\circ} \mathrm{C}, y_{\wedge}$ germination at $5{ }^{\circ} \mathrm{C}$. $y_{\bullet} \operatorname{IVG}(\%)$ at $20^{\circ} \mathrm{C}, y_{\wedge} \operatorname{IVG}(\%)$ at $5{ }^{\circ} \mathrm{C}$. 


\section{REFERÊNCIAS BIBLIOGRÁFICAS}

BANZATTO, D.A.; KRONKA, S.N. Experimentação agrícola. Jaboticabal: FUNEP, 1989. 247p.

BORGES, E.E.L.; CASTRO, J.L.D.; BORGES, R.C.G. Alterações fisiológicas em sementes de jacaré (Piptadenia communis) submetidas ao envelhecimento precoce. Revista Brasileira de Sementes, v. 14, n.1, p.9-12, 1992.

BRACCINI, A. L.; BRACCINI, M.C.L.; SCAPIM,C.A. Mecanismos de deterioração das sementes: aspectos bioquímicos e fisiológicos. Informativo ABRATES, v.11,n.1,p.10-15, 2001.

BRASIL. Ministério da Agricultura e Reforma Agrária. Regras para análise de sementes. Brasília: DNPV - DISEM, 1992. 365p.

CARneiro, J.G.A. Armazenamento de sementes florestais. Curitiba: FUPEF, 1985. 35p. (Série Técnica, 14).

FIGLIOLIA, M.B. Conservação de sementes de essências nativas. Boletim Técnico do Instituto Florestal, n.42, p. 1-18, 1988.

FIGLIOLIA, M.B. et al. Efeito do acondicionamento e do ambiente de armazenamento na conservação de sementes de sibipiruna. Revista Brasileira Horticultura Ornamental, v. 7, n.1, p.57-62, 2001.

FIRMINO, J. L.; SANTOS, D.S.B.; SANTOS, B.G. Utilização de alguns testes de viabilidade e vigor e composição química em sementes de cerejeira (Amburana acrena (Ducke) A. C. Smith).

Revista Árvore, v. 19, n.3, p.286-292, 1995.

FREITAS, R.A. et al. Storability of cotton seeds prected by vigour test. Seed Science and Technology, v. 30, p.403- 410, 2001.

GARCIA, S.L.R. Curso de estatística experimental. Viçosa, MG: Faculdade de Viçosa, 2001. p. 227- 233.
GEMAQUE, R.C.R. Maturação, tolerância à dessecação e alterações na qualidade de sementes de ipê-roxo (Tabebuia impetiginosa (Mart.) Standl.) envelhecidas artificialmente. 1999. 93f. Dissertação (Mestrado em Ciências Florestais) Universidade Federal de Lavras, Lavras, 1999.

LEÓN-LOBOS, P.; ELLIS, R.H. Seed storage behaviour of Fagus sylvatica and Fagus crenata. Seed Science Research, v.12, p. 31-37, 2002.

LORENZI, H. Caesalpinia peltophoroides Benth. In: LORENZI, H. Árvores brasileiras: manual de identificação e cultivo de plantas arbóreas nativas do Brasil. Nova Odessa: Plantarum, 1992. p. 148.

MARCOS FILHO, J. Teste de envelhecimento acelerado. In: VIEIRA, R.D.; CARVALHO,N.M. Testes de vigor em sementes. Jaboticabal: FUNEP, 1994. p. 133-150.

MAGUIRE, J.D. Speed of germination-aid in selection and evaluation for seedling emergence and vigor. Crop Science, v.2, n. 1, p. 176-177, 1962.

MATTHEWS, S. Physiology of seed ageing. Outlook on Agriculture, v. 14, n.2, p.19-23, 1985.

NODARI, R.O. et al. Conservação de frutos e sementes de palmiteiro (Euterpe edulis Matius) sob diferentes condições de armazenamento. Revista Árvore, v.22, n.1, p.1-10, 1998.

STATSOFT, INC. Statistica for Windows (Computer program manual) Tulsa, OK: StatSoft, 2000. Inc 2300E ast 14 th Street, Tulsa, OK 74104.

VARELA, V.P.; FAÇANHA, J.G.V. Secagem de sementes de cumaru: influência sobre a germinação e vigor. Pesquisa Agropecuária Brasileira, v.22, n.9/10, p. 959-963, 1987.

WOODSTOCK, L.W. Physiological and biochemical test for seed vigor. Seed Science and Technology, v.1, p. 127-57, 1973. 